\title{
The Acquisition of the Thematic Roles of the English Verb Open by College Students
}

\author{
Namkil Kang and Hisashi Morita \\ Far East University/Aichi Prefectural University \\ kangnamkil@kdu.ac.kr/hmorita@for.aichi-pu.ac.jp
}

\begin{abstract}
The main purpose of this paper is to provide an analysis of the acquisition of the English verb open and its thematic roles in L2 English by Korean learners. First, the Koreanlearners in the study did not give support to the subject hierarchies of Larson (1988), Grimshaw (1990), Dowty (1991), and Saeed (2009) and exhibited the following order in the experiment of the thematic roles of open and yelta 'open': Agent Goal > Instrument. We have contended that the learners' subject hierarchy (agent $>$ goal > instrument) should be represented in the core grammar since it comes from the learners cognition and it is supported by the synchronic computational system (the British National Corpus). Second, the results of our experiment clearly illustrate the fact that the Korean learners do entertain the hypothesis that learners look for similarities whenever they can find them, but they do not respect Chomsky's UG theory since the acquisition of English thematic roles by the Korean learners takes place through positive transfer and negative transfer of their native thematic roles. Third, we have argued in this paper that when all three thematic roles (agent, instrument, and goal) appear within sentence, the Korean learners judged the order of English thematic roles in accordance with their D1, namely transfer, since the orders of the three thematic roles that the English verb open allows are a subset of the orders that the Korean verb yelta 'open' allows (the Superset Principle). Additionally, we have claimed that our subject hierarchy captures the order of acquisition of subject functions and reflects the degree of markedness which correlates with the order of acquisition. Finally, we have maintained that agen subjects and goal subjects are absolutely universal subject functions which are governed by UG, whereas instrument subjects are subject functions that lie outside $U G$.
\end{abstract}

Keywords: open, Chematic roles, agent, goal, instrument, EFL

\section{Introduction}

It has been noticed in the literature (Fillmore (1968), Givón (1984), Dowty (1991), Saeed (2009) and Ramchand (2010)) that the process of different thematic roles occupying the subject position is a hierarchical process:

(1) Agent $>$ Recipient/ Benefactive $>$ Theme/Patient $>$ Instrument $>$ Location

(Saeed (2009))

(2) Agent $>$ Instrument/Experiencer $>$ Patient $>$ Source/Goal

(Dowty (1991))

As noted by Saeed (2009), the leftmost thematic roles in (1) and (2) are the preferred and expected subjects, while the rightmost thematic roles are less expected subjects. 
The ultimate goal of this paper is to provide an analysis of the acquisition of the English verb open in L2 English by Korean learners and to investigate Korean learners' knowledge of the thematic roles of the English verb open. This paper is organized as follows. In Section 2, we discuss theoretical assumptions about the subject hierarchies proposed by Dowty (1991) and Saeed (2009). Section 3 provides background discussion regarding the four main goals of our experiment and subjects. In Section 4, our discussion centers on analyzing the acquisition of the thematic roles of the English verb open by the Korean learners. Section 5 is devoted to presenting a more detailed analysis relating to the acquisition of the thematic roles of the English verb open.

\section{A Subject Hierarchy: Previous Analyses}

With regard to a subject hierarchy which is the process of different thematic roles occupying the subject position, there have been several versions in the literature, e.g Fillmore (1968), Givón (1984), Larson (1988), Grimshaw (1990), Dowty (1991), Saeed (2009), and Ramchand (2010).

The idea that there are stronger and weaker candidates for the subject position has been suggested by Dowty (1991). His version of a subject hierarchy is as in (3):

(3) Agent $>$ Instrument/Experiencer $>$ Patient $>$ Source/Coal

In order to account for (3), Dowty (1991) suggest that there are two basic prototypes: ProtoAgent and Proto-Patient:

(4) Properties of the Agent Proto-Role

a. volitional involvement in the event or state

b. sentience (and/or perception)

c. causing an event or change of state in another participant

d. movement (relative to the position of another participant)

(5) Properties of the Patient Proto-Role

a. undergôes change of state

b. incremental theme

c. causally affected by another participant

d. stationary relative to movement of another participant

Dowty's prototype and entailments approach is embodied by an argument selection principle, as indicated by (6):

(6) Argument Selection Principle: In predicates with grammatical subject and object, the argument for which the predicate entails the greatest number of Proto-Agent properties will be lex calized as the subject of the predicate; the argument having the greatest number of Proto-patient entailments will be lexicalized as the direct object.

Now consider (7), taken from Saeed (2009):

(7) a. Captain Nemo sank the ship with a torpedo.

b. The torpedo sank the ship.

c. The ship sank.

(Saeed 2009) 
As argued in Saeed (2009), Captain Nemo in (7a) can appear in the subject position since Captain Nemo has volition, sentience, causation, and movement. The torpedo in (7b) can also appear in the subject position since it has causation and movement. Saeed (2009) points out that even though the ship in (7c) has only movement, for it to be a subject is enough. This fact thus gives further weight to the claim that there are stronger and weaker candidates for the subject position.

The claim that there are strong and weaker candidates for the subject position is echoed by Saeed (2009). Consider the following sentences:

(8) Ursula broke the ice with a pickaxe.

(9) The pickaxe broke the ice.

(10) The ice broke.

(Saeed 2009)

As plausibly argued in Saeed (2009), Ursula in (8) is both agent and subject, the ice is both patient and direct object, and the pickaxe is the instrument In (9) the agent does not occur in the subject position and the instrument (the pickaxe) appears in the subject position. In (10), with no agent or instrument occurring in the subject position, the patient appears in the subject position. Saeed (2009) maintains that "his process of different roles occupying the subject position is a hierarchical process not onty in English but across many languages" (Saeed 2009). Saeed (2009) further maintains that When speakers are constructing a sentence, they tend to place an agent mto subject position, the next preference being for a recipient or benefactive, then themetpatient, then other roles" (Saeed 2009). Consequently, Saeed (2009) constructs the following aniversal subject hierarchy:

(11) Agent $>$ Recipient/Benefactive $>$ Theme/Patient $>$ Instrument $>$ Location

Saeed (2009) claim that this hierarchy can be read in the following way: "the leftmost elements are the prefered, most basjc and expected subjects, while moving rightward along the string gives us less expected subjects" (Saeed 2009).

\section{Method}

\subsection{The Main Goals of Experiments}

Our experiment had four main goals. The first was to provide an answer to the question of whether or notKorean learners of English give support to Larson (1988), Grimshaw (1990), Dowty (1991) and Saeed (2009). The second goal was to provide an answer to the following question: Does learning take place through positive transfer and negative transfer of native thematic roles? Third, when all three thematic roles (agent, instrument, and goal) appear within a sentence, in what order do these thematic roles appear? Can the L2 learners capture the order? The fourth was to provide answers to the following questions: Does the learners' subject hierarchy predict the order of acquisition of subject functions? Does the learners' subject hierarchy reflect the degree of markedness which correlates with the order of acquisition?

\subsection{Subjects}


Twenty Korean EFL college students whose ages ranged from 21 to 27 participated in our experiment. These adult subjects were undergraduate students of a private university in the Chungbuk Province in Korea. Only the adult subjects who successfully responded to a preliminary test were included in the main experiment. We asked 20 Korean students whether or not 9 target sentences containing the English verb open and 11 sentences containing the Korean verb yelta 'open' are grammatical. No feedback was given to the adult learners on their performance during our experiment.

\section{Results}

First, we included (12) and (13) in our experiment to assess adults' knowledge of agent subjects and instrument subjects:

(12) a. The boy opened the door. b. *The boy opened.

(13) a. The key opened the door.

b. Ku ki-ka ku mwun-ul yel-ess-ta. the key-NOM the door-ACC open-Past-DEC (The key opened the door.)

As already addressed, a major question with regard to sentences like (12) and (13) is whether or not the Korean learners of Engtishlend suppont to the subject hierarchies proposed by Dowty (1991) and Saeed (2009)? To begin with, the Korean adults showed a level of perfection (close to 100\%) in the experiment of (12a). That is, the adults' correct responses to (12a) were $95 \%$, whereas the adults incorrect cesponses to (12a) were 5\%. It must be noted, however, that the adults' incorrect responses to (13a) were about $60 \%$. The fact that the adults' incorrect responses to (13a) were $60 \%$ suggests that instrument subjects are not preferred in comparison to agent subjects. The same can be said about Korean sentence (13b) corresponding to (13a). That is the adults' incorrect responses to the Korean sentence (13b) were $60 \%$. The results of this experiment indicate that these Korean adults show a strong preference toward the use of the agent as a subject, which implies that they completely acquired the property associated with the function of the agent in the sentence. A crucial point we must address here is that the agent as a subject is used in (12b), but the sentence is ungrammatical and the adults' incorrect responses to the sentence were $45 \%$. We may take this fact as indicating that even though they did not know the fact that objects cannot be omitted after transitive verbs, the Korean adults showed a strong preference toward the use of the agent as a subject. Again, it is interesting to note that in (12a) and (12b) the Korean adults do respecthe fact that agents are the preferred, most basic, and expected subjects. Then the acquisition of the function of the agent in L2 English by the Korean learners may be the reflection of (1) and (2). A further point we must address here is that the L2 learners' incorrect responses to (13a) and (13b) were the same (60\%), which suggests that they may be unwittingly relying on Korean thematic roles and extending them to create English thematic roles through negative transfer. Ellis (2012) argues that where L1 and L2 are identical, learning can take place easily through positive transfer of the native-language pattern, but where they are different, learning difficulty arises and errors resulting from negative transfer are likely to occur. Consequently, the analysis of the Korean adults' responses reveals that instrument subjects are not preferred over agent subjects. 
There is a significant difference in performance between the sentence containing instrument subjects and the sentence containing goal subjects:

(14) a. The door opened.

b. ku mwun-i yel-lyess-ta.

the door-NOM open-Past-DEC

(The door opened.)

The English sentence (14a), which contains a goal subject, is grammatical. The adults' correct responses to (14a) were $60 \%$, whereas their correct responses to the Korean sentence (14b) corresponding to (14a) were 80\%. As observed earlier, in the experiment with instrument subjects, learning difficulty arose and errors resulting from negative transfer occurred. If we consider this, the fact that the adults' correct responses to (14a) were $60 \%$ implies that the acquisition of goal subjects in L2 English by the Korean learners may be affected by positive transfer. Also, the results of this experiment reveal that goal subjects in English are preferred over instrument subjects, but they are not preferred over agent subjects.

Unlike (13a) and (14a), the following sentences which contain an instrument subject and a goal subject, respectively, are ungrammatical since, as Palmer (1987) points out, there is no goal, despite the fact that the goal must always be present:

(15) *The key opened.

(16) *The boy opened.

The Korean learners' correct esponses to (15) were $75 \%$, whereas their incorrect responses to (15) were $25 \%$. On the other hand the adults' correct responses to (16) were $45 \%$, whereas their incorrect responses to (16) were 55\%. The Korean adults' poor performance on sentence (15) and sentence (16) might be attributed to incomplete learning of the thematic roles of the English verb open. That is, it cannot be denied that 25 percent of the adult learners with regard to (15) and 55 percent of the adult learners regarding (16) did not perceive that the goal must always be present. However, we can see a significant difference in performance between the sentence containing the instrument subject and the sentence containing the agent subject. L is perhaps worth emphasizing that agent subjects are preferred by the Korean adults over instrument subjects since 25 percent of the Korean adults thought of (15) as grammatical, whereas 55 percent of them thought of (16) as grammatical.

As observed in-(13) and (14), the fact that goal subjects are preferred over instrument subjects is further supported by the following sentences which were used in our experiment:

(17) *The door opened by the boy.

(18) *The key opened the door by the boy.

The adults' incorrect responses to (17) were 30\%, whereas their incorrect responses to (18) were $25 \%$. That is, 30 percent of the L2 learners thought of (17) as grammatical even though it is ungrammatical, whereas 25 percent of them thought of (18) as grammatical. Thus, the above sentences which were used in our experiment seem to crucially show that goal subjects are preferred by L2 learners over instrument subjects. These observations lead us to conclude that the acquisition of English thematic roles in L2 English by the Korean learners is not affected by the subject hierarchies of Dowty (1991) and Saeed (2009) since goal subjects in 
English are preferred over instrument subjects, but they are not preferred over agent subjects. Thus, unlike (1) and (2), the Korean L2 learners allow the following order in the experiment of the thematic roles of the English verb open:

\section{(19) Agent > Goal > Instrument}

Most importantly, (19) also holds for Korean since 90 percent of the Korean adult learners chose agent subjects in our experiment, 80 percent of them chose goal subjects, and 40 percent of them chose instrument subjects:

(20) Ku sonyen-i ku mwun-ul yel-ess-ta. the boy-NOM the door-ACC open-Past-DEC (The boy opened the door.)

(21) Ku mwun-i yel-lyess-ta. the door-NOM open-Past-DEC (The door opened.)

(22) Ku ki-ka ku mwun-ul yel-ess-ta. the key-NOM the door-ACC open-Past-DEC (The key opened the door.)

Our experiment indicates that the adults perrormed better on the agent subject than on the instrument subject or the goal subject. The adults' performance on the agent subject was strikingly higher than performances on the instrument subject since the Korean learners' correct responses to (22) were $40 \%$, whereas the Korean adults showed a level of perfection $(90 \%)$ with respect to (20). In the case of (21), the Korean learners' correct responses to it were $80 \%$ and the adults' performance on (21) was higher than performances on (22). On the basis of this finding, we may conclude that the Korean adults show a strong preference toward the use of the agent as a subject and that the results of our experiment on thematic roles which the Korean verb yelta 'open admits, also argue for (19).

Similarly, in (23), (24), and (25), 95 percent of the Korean adults thought of (23) as grammatical, 60 percent of them thought of (24) as grammatical, and 40 percent of them thought of (25) as grammatical. This amounts to saying that 95 percent of the Korean adults chose agent subjects, 60 percent of them chose goal subjects, and 40 percent of them chose instrument subjects

(23) The boy opened the door.

(24) The door opened.

(25) The key opened the door.

In our experiment, the Korean learners, with respect to the English sentences including (23), (24), and (25) and the Korean sentences including (20), (21), and (22), exhibited almost identical responses. That is, in Korean and English, goal subjects are preferred over instrument subjects, but they are not preferred over agent subjects. Thus, the results of our experiment clearly illustrate the fact that in the case of the acquisition of the English verb open, learning takes place through positive transfer and negative transfer of native thematic 
roles. Ringbom (2007) emphasizes that learners do not look for differences; they look for similarities whenever they can find them. On the other hand, Ellis (2012) argues that "UG theories of L2 acquisition differ in the role they attribute to the L1" (Ellis 2012). That is, "learners have complete access to Universal Grammar and thus do not need to rely on their L1-a zero transfer position" (Ellis 2012). Clearly, the results from our Korean adults in our experiment do entertain the hypothesis that learners look for similarities whenever they can find them, but they do not respect the UG theory that learners have complete access to Universal Grammar (Chomsky 1981, 1986, 1989, 1995, 2000, 2001, 2013) and thus do not need to rely on their L1 (a zero transfer position).

The third goal of our experiment was to provide an answer to the following question: when all three thematic roles (agent, instrument, and goal) appear within a sentence, in what order do these thematic roles appear? How can the L2 learners capture the order? Consider the following sentences which were used in this experiment:

(26) a. The boy opened the door with the key.

b. ku sonyen-i ku mwun-ul ku kilo yel-ess-ta. The boy-NOM the door-ACC the key with open-Past-DEC) (The boy opened the door with the key.)

c. ku sonyen-i ku kilo ku mwun-ul yel-ess-ta.

The boy-NOM the key with the door (The boy opened the door with the key.)

When all three thematic roles (agent, instrument, andgoal) appear within a sentence, the order of the thematic roles of the Korean verb yelta open' is flexible, as illustrated in (26b) and (26c), but that of the thematic roles of the English verb open is rigid, as indicated in (26a). That is, the English verb open allows the fixed order of agent-goal-instrument, whereas the Korean verb yelta 'open' aliows two different orders: agent-instrument-goal and agentgoal-instrument. The Koreanlearners' correct responses to (26a) were $85 \%$ and their correct responses to (26b) were $75 \%$. On the otherhand, they showed a level of perfection $(95 \%)$ in the experiment for (26c). Note, however, that the following sentences are also grammatical even though we difhot experiment them:

(27) a. With the key the boy opened the door.

b. ku kilo ku sonyen-i ku mwun-ul yel-ess-ta. the key with the-bo-NOM the door-ACC open-Past-DEC (The boy opened the door with the key.)

As illustrated in (27), both English and Korean allow another order of thematic roles associated with open and yelta 'open' and more are possible in the case of Korean. As indicated in (26a), (26b), (26c), and (27), English allows two orders, but Korean allows several different orders and the Korean learners prefer agent-instrument-goal to agent-goalinstrument. We wish to argue that since the orders of three thematic roles that the English verb open allows belong to the orders that the Korean verb yelta 'open' allows, the Korean learners of English judged (26a) in accordance with their L1, namely transfer. Our assumption is further supported by the following sentences which were used in this experiment:

(28) a. *The key opened the door by the boy.

b. *ku ki-ka ku mwun-ul ku sonyen uyhay yel-ess-ta. 
the key-NOM the door-ACC the boy by open-Past-DEC (The key opened the door by the boy.)

As indicated in (28), both English and Korean do not allow the order of instrument-goalagent. The Korean learners' correct responses to (28a) and (28b) were $75 \%$ and $90 \%$, respectively, which in turn suggests that L2 learners acquired the order of thematic roles associated with the English verb open. Again, we assume that that the Korean learners of English did not look for differences. They looked for similarities between English and Korean. Thus, the acquisition of thematic roles in L2 English by the Korean learners may be affected by transfer.

Now consider (29) and (30):

(29) a. *The door opened with a key.

b. ku mwun-i kilo yel-lyess-ta. the door-NOM key with open-Past-DEC (The door opened with a key.)

(30) a. *The door opened by the boy

b. ku mwun-i ku sonyen uyhay yel-lyess-ta.

the door-NOM the boy by open-Past-DEC

(The door opened by the boy.)

In the case of English, the goal can be-subject only if the other two (instrument or agent) are absent. Since the instrument and the agent in (29a) and (30a) occur with the goal, (29a) and (30a) are ungrammatical. Note, however, that the Korean sentences (29b) and (30b) corresponding to (29a) and (30a) are grammatical even though the instrument and the agent appear with the goal. Most importantly, the Korean learners' incorrect responses to (29a) and (30a) were $40 \%$ and $30 \%$, espectively. On the other hand, their incorrect responses to (29b) and (30b) were $20 \%$ and $0 \%$. An immediate question is "why are the Korean learners" incorrect responses to (29a) and (30a) so high? The reason may be because the English sentences (29a) and (30a) are marked in comparison with the Korean sentences (29b) and (30b). Unlike Korean, English allows the goal to appear in the subject position only if the other two (instrument or agent) are absent. Ellis (2012) argues that "unmarked structures are those that are common in the world's language" (Ellis 2012). That is, he argues that in Chomskyan theory, "unmarked structures are those that are governed by UG, whereas marked structures are those that lie outside UG" (Ellis 2012). One of a number of hypotheses relating to markedness is that "learners acquire less marked structures before more marked ones" (Ellis 2012). Now a question to be asked here is "does the learners' subject hierarchy (19) capture the order of acquisition of subject functions and reflect the degree of markedness which correlates with the order of acquisition?" Consider the following sentences, repeated here

(31) a. The door opened.

b. The boy opened the door.

c. The key opened the door.

As observed earlier, there was a significant difference in performance among the sentence containing the goal, the sentence containing the agent, and the sentence containing the instrument. That is, the adults' incorrect responses to (31a), (31b), and (31c) were 40\%, 5\%, 
and $60 \%$, respectively. The Korean learners' incorrect responses to (31a), (31b) and (31c) seem to crucially show that they acquired unmarked subject functions (agent subject and goal subject), but they did not acquire marked subject functions (instrument subject). Then the adult learners' subject hierarchy (19) captures the order of acquisition of subject functions and reflects the degree of markedness which correlates with the order of acquisition. This fact leads us to conclude that L2 learners may acquire agent subject functions first, then goal subject ones, then instrument subject ones. However, we must be sure whether the order of acquisition is influenced by some other factor other than markedness. Ellis (2012) argues that "learners are more likely to acquire a frequent but marked structure before an infrequent but unmarked structure than vice versa" (Ellis 2012). We take this fact as indicating that agent subjects are the most frequent in the input and goal subjects are more frequent than instrument subjects. Finally, a crucial point we must address here is that from the viewpoint of Chomskyan syntax, unmarked subject functions, such as agent subjects and goal subjects, may be those that are governed by UG, whereas marked subject functions such-as histrument subjects may be those that lie outside UG.

\section{Discussion}

Now we move onto the question of whether or not the Korean learners of English give support to the subject hierarchies proposed by Larson (1988) and Grimshaw (1990). Consider (32), (33), and (34), taken from Ramchand (2010):

(32) Larson (1988)

Agent $<$ Theme $<$ Goal < Obliques (manner, location, time)

(33) Grimshaw (1990)

Agent $<$ Experiencer $<$ Goal/Source Locătion? Theme

(34) Principle of Argument Realization M(Larson 1988)

If $a$ is a predicate and $B$ is an argument of $a$, then $\beta$

must be realized within a projection headed by $a$.

Principle of Argument Realization 2 (Larson 1988)

If a verb a determines $\Theta$-roles $\Theta 1, \Theta 2 \ldots \Theta \mathrm{n}$, then

the lowest role on the Thematic Hierarchy is assigned to

the lowest argument in constituent structure, the next

lowest argumênt, and so on.

It is important to note that there has not been consensus on the exact nature of the subject hierarchy as evidenced by (3), (11), (32), and (33) and that the adult learners' hierarchy (agent $>$ goal $>$ instrument) supports neither Larson's (1988) subject hierarchy nor Grimshaw's (1990) subject hierarchy. It is argued in Ramchand (2010) that Dowty's (1991) “principle of argument selection cannot be seen as a fact about the synchronic computational system since decisions about what gets to the subject are not computed on-line" (Ramchand 2010). It is also argued in Ramchand (2010) that "Dowty's principle basically gives up the idea that the generalizations we see should be represented in the core grammar" (Ramchand 2010). Consequently, the results from the learners' responses (agent $>$ goal $>$ instrument) do not support the subject hierarchies proposed by Larson (1988) and Grimshaw (1990), to say nothing of the subject hierarchies proposed by Dowty (1991) and Saeed (2009). Likewise, the 
British National Corpus does not back up the hierarchies proposed by Larson (1988), Grimshaw (1990), Dowty (1991), and Saeed (2009). It is interesting to point out, however, that the British National Corpus supports the adult learners' subject hierarchy (agent > goal > instrument). Table 1 shows the use and frequencies of the English verb open in the British National Corpus:

\section{Table 1 The Frequencies of the English Verb open in the British National} Corpus

\begin{tabular}{|c|c|c|}
\hline Subjects & Frequency & Percentage \\
\hline Agent subject & 1301 & 79.8 \\
\hline Goal subject (the door) & 328 & 20.1 \\
\hline $\begin{array}{c}\text { Instrument subject (the } \\
\text { key) }\end{array}$ & 0 & 0 \\
\hline Total & 1629 & 100 \\
\hline
\end{tabular}

The number of the tokens of agent subjects, goal subjects, and instrument subjects of the English verb open in the British National Corpus is 1629. One characteristic of subject frequency analyses in the British National Corpus is that the agent subject has the highest frequency (1301 tokens) and the highest proportion (79.8\%). Although the overall frequency of the goal subject (the door) is quite low (328 tokens), it is one of the primary subjects of the English verb open with agent subjects. On the other hand, the frequency of the instrument subject (the key) in the British National Corpus is 0 even though it is one of the subjects of the English verb open. A crucial pointwe must address bere is that the results from table 1 and the adult learners' responses seem to suggest that the learners' subject hierarchy (agent > goal > instrument) should be represented in the core grammar since it comes from the learners' cognition and it is supported by the synchronic computational system (the British National Corpus). That is, decisions about what gets to be the subject are computed in terms of the learners' perception and the frequencies of the English verb open from the British National Corpus. A further point we wish to mention here is that the results from both table 1 and the Korean learners' responses do confirm that agent subjects are the preferred, most basic, and expected subjects. Also, it is important to note that the fact that goal subjects are preferred by the Korean learners over instrument subjects is further supported by table 1 since the frequencies of the god subject and those of the instrument subject in the British National Corpus are 328 and 0, respectively. Consequently, both learners' subject hierarchy and table 1 predict that when speakers construct a sentence, they will place an agent into the subject position, then a goal then an instrument. Thus, we take this as indicating that the learners' hierarchy (agent > goal > instrument) may be a universal subject hierarchy and thus should be represented in the core grammar.

Now we turn to the second question: Does learning take place through positive transfer and negative tuansfer of native thematic roles? Consider (35) and (36), repeated below:

(35) a. Ku sonyen-i ku mwun-ul yel-ess-ta. the boy-NOM the door-ACC open-Past-DEC (The boy opened the door.)

b. Ku mwun-i yel-lyess-ta. the door-NOM open-Past-DEC (The door opened.)

c. Ku ki-ka ku mwun-ul yel-ess-ta. the key-NOM the door-ACC open-Past-DEC 
(The key opened the door.)

(36) a. The boy opened the door.

b. The door opened.

c. The key opened the door.

In (35), 90 percent of the Korean learners chose agent subjects, 80 percent of them chose goal subjects and 40 percent of them chose instrument subjects. On the other hand, in (36), 95 percent of the Korean adults thought of (36a) as grammatical, 60 percent of them though of (36b) as grammatical, and 40 percent of them thought of (36c) as grammatical. This amounts to saying that 95 percent of them chose agent subjects, 60 percent of them chose goal subjects, and 40 percent of them chose instrument subjects. The following figures show the percentages of the learners' correct responses to agent subjects, goal subjects, and instrument subjects:

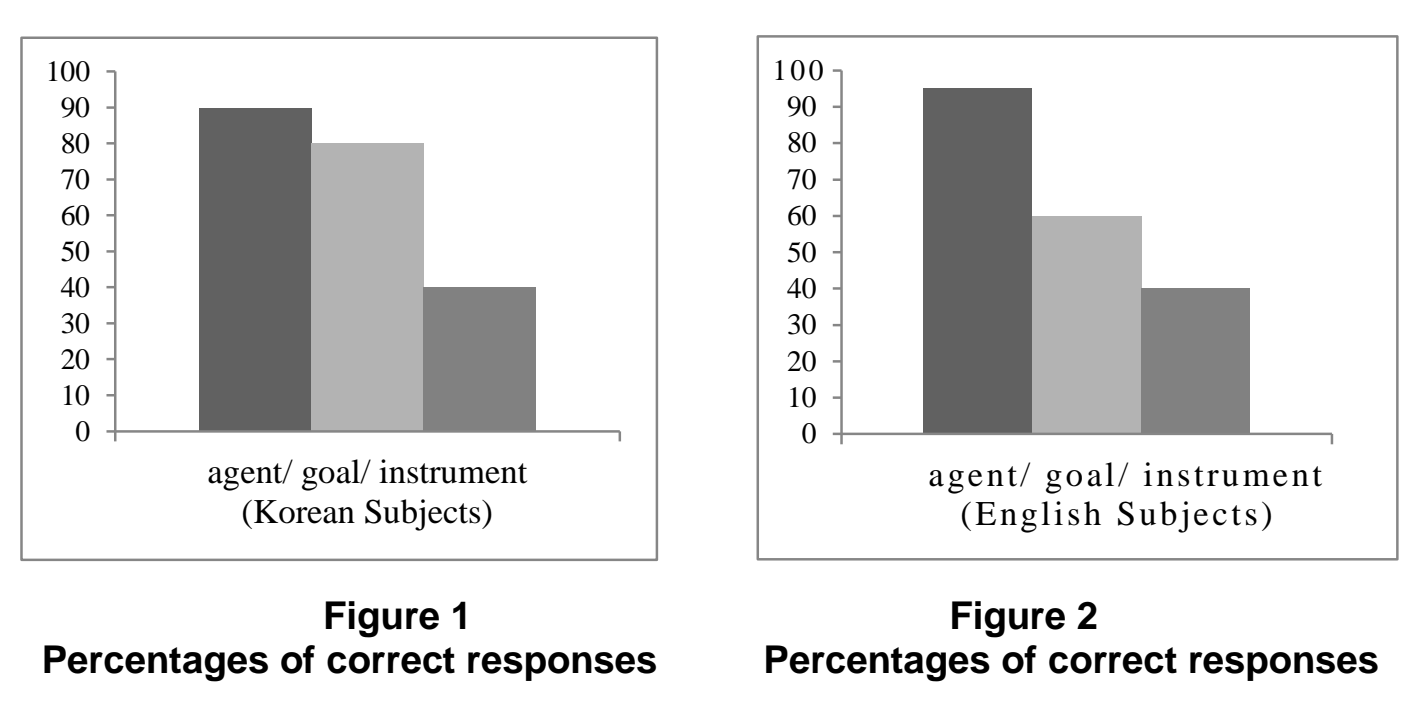

Figure 1 and Figure 2 indicate that the adults performed better on agent subjects than on goal subjects and instrument subjects. In Figure 1 and Figure 2, the learners' performance on Korean agent subjects/English agent subjects was strikingly higher than performances on Korean goal subjects/English goal subjects and Korean instrument subjects/English instrument subjects. Also, it is interesting to point out that as indicated in Figure 1 and Figure 2, the Korean learners showed a preference toward agent subjects and goal subjects, but they did not show any preference for instrument subjects.

Now an mmediate question is "why are the Korean learners' responses to (36a), (36b), and (36c) and their responses to (35a), (35b), and (35c) alike?" We claim that the reason why their responses to Korean sentences and English sentences are alike may be because the acquisition of (36) by the Korean learners took place through facilitation (positive transfer). As plausibly argued in Ellis (2012), "the central claim of the Contrastive Analysis Hypothesis was that differences between the target language and the L1 resulted in learning difficulty and similarities between the target language and the L1 resulted in learning ease" (Ellis 2012). Clearly, our claim is not compatible with the Contrastive Analysis Hypothesis since the Korean learners' L1 facilitated L2 learning, despite the fact that the syntactic structure of Korean and that of English are completely different. However, Ringbom (2007) emphasized that learners look for similarities whenever they can find them. Ellis (2012) assumes with 
Ringbom (2007) that transfer can take place as a result of both similarity and difference and that it is similarity that is the more important. More interestingly, Ellis (2012) assumes with Schachter (1983) that "transfer can be viewed as a source of information that learners can use to construct and reconstruct hypotheses by means of inductive inferencing (scanning data, observing regularities, and generalizing) and deductive inferencing (testing hypotheses by looking in the first instance for confirming evidence and subsequently for disconfirming evidence)" (Ellis 2012). Given the preceding discussion, we can reasonably propose that the reason why the adult learners' responses to Korean sentences (35a, 35b, and 35c) and English sentences (36a, 36b, and 36c) are alike is because the acquisition of (36a), (36b), and (36c) by the Korean learners took place through facilitation (positive transfer).

The third goal of our experiment was to provide an answer to the following question: When all three thematic roles (agent, instrument, and goal) appear within a sentence, in what order do these thematic roles appear? How can the L2 learners capture the order? Consider the following sentences, repeated below:

(37) a. The boy opened the door with the key.

b. With the key the boy opened the door.

c. *The boy opened with the key the door.

(38) a. ku sonyen-i ku mwun-ul ku kilo yel-ess-ta. The boy-NOM the door-ACC the key with open-Past-DEC (The boy opened the door with the key.
b. ku sonyen-i ku kilo
ku mwun-ul yel-ess-tas.

The boy-NOM the key with the door open Past-DEC

(The boy opened the door with The key.)

c. ku kilo ku sonyen-i ku mwun-ul gel-ess-ta. the key with the boy-NOM the door-ACC open-Past-DEC

(The boy opened the door with the key.)

d. ku mwun-ul ku onyen-i kukilo yel-ess-ta.

the door-ACC the boy-NOM the key with open-Past-DEC

(The boy opened the door with the key.)

As indicated in (37a) and (37b), English allows only two orders (agent-goal-instrument and instrument-agent-goal) and hus does not allow the order of agent-instrument-goal, as in (37c). On the other hand, Korean allows free orders (agent-goal-instrument, agent-instrumentgoal, instrument-agent-goal, and goal-agent-instrument), as in (38). It is pointed out in Berwick (1985) and Ellis (2012) that "if learners construct a conservative grammar, Y, which generates a subset of the sentences generated by some other grammar, $\mathrm{X}$, this grammar can subsequently be expended on the basis of positive evidence" (Ellis 2012). It is also argued in Ellis (2012) that "if learners begin with a superset grammar, $X$, they would require negative evidence lo narrow its scope in order to construct Y" (Ellis 2012). If this theory works for Korean and English, Korean is a superset grammar since it allows free orders, as indicated in (38), while English is a subset grammar of Korean since English allows only two orders, as illustrated in (37). It thus seems reasonable to understand that since Korean is a superset grammar of English, L1 facilitated L2 learning. That is, the acquisition of the order of English thematic roles by the Korean learners took place through the Superset Principle, which requires negative evidence to narrow its scope in order to construct a subset grammar. Note that a superset grammar allows the order of (38b), but a subset grammar does not allow that 
of (37c). Accordingly, for learners to construct a subset grammar, they require negative evidence such as (37c).

Finally, consider (39), repeated here:

(39) a. The door opened.

b. The boy opened the door.

c. The key opened the door.

Crystal (1996) argues that "an unmarked property is one which accords with the general tendencies found in all languages; a marked property is one which goes against these general tendencies" (Crystal 1996). Similarly, Ellis (2012) argues that markedness "refers to the general idea that some structures are more natural or basic than other structures" (Ellis 2012). He argues that "in Chomskyan linguistics, unmarked structures are those that are governed by UG and which, therefore, require only minimal evidence for acquisition" (Ellis 2012). On the other hand, he argues that "marked structures are those that lie outside UG" (Ellis 2012). Crystal (1996) assumes with Radford (1981) that "a relative" universal is one which characterizes a general tendency in a language, and allows for exceptions" (Cystal 1996). On the other hand, he argues that "an absolute universal is one which characterizes all languages, without exception" (Crystal 1996). Also, Chomsky (2013) argues that "the simpler UG, the greater the hope that evolution of language might someday be at least partially understood" (Chomsky 2013). Thus, if UG (Chomsky 2013) Is to be simpler, it must reflect only the general tendencies found in all languages. Notice, now, that there was a significant difference in performance among the sentence containing the god the sentence containing the agent, and the sentence containing the instrument That is, the adults' incorrect responses to $(39 \mathrm{a})$, $(39 b)$, and (39c) were $40 \%, 5 \%$, an $160 \%$, respectively, which seems to crucially show that agent subjects and goal subjects are unmarked subject functions, but instrument subjects are marked subject functions. Thus, agent subjects and goal subjects are absolutely universal subject functions which are governed by $\mathbb{U G}$, whereas instrument subjects are subject functions that lie outside UG. Then the learners' subject hierarchy (agent > goal > instrument) captures the order of acquisition of subject functions and reflects the degree of markedness which correlates with the order of acquisition. That is, L2 learners will acquire unmarked agent subject functions first then unmarked goal subject ones, then marked instrument subject ones since agent subjects and goal subjects are absolutely universal subject functions which are governed by UG (agent subjects and goal subjects require only minimal evidence for acquisition), but instrument subjects are subject functions that have none of the general tendencies found in als languages (thus, they lie outside UG).

\section{Conclusion}

To sum up, first, we have provided an answer to the question of whether or not the Korean learners of English lend support to Larson (1988), Grimshaw (1990), Dowty (1991) and Saeed (2009). The Korean learners in the study did not give support to the subject hierarchies proposed by Larson (1988), Grimshaw (1990), Dowty (1991), and Saeed (2009) and exhibited the following order in the experiment of the thematic roles of open and yelta 'open': Agent > Goal > Instrument. We have suggested that the adults' subject hierarchy (agent > goal > instrument) should be represented in the core grammar since it comes from the learners' cognition and it is supported empirically by the British National Corpus. Second, we have claimed that the results from the Korean adults in our experiment argue for the hypothesis that learners look for similarities whenever they find them, but they do not entertain Chomsky's UG theory since the acquisition of English thematic roles by the Korean 
learners takes place through positive transfer and negative transfer of their native thematic roles. Third, we have claimed that the acquisition of the order of English thematic roles by the Korean learners took place through the Superset Principle since Korean is a superset grammar when compared to English. Finally, we have maintained that agent subjects and goal subjects are absolutely universal subject functions which are governed by UG, whereas instrument subjects are subject functions that lie outside UG. This is motivated by the fact that the learners' subject hierarchy captures the order of acquisition of subject functions and reflects the degree of markedness which correlates with the order of acquisition.

\section{Acknowledgements}

This article is a revised and expanded version of a paper entitled [The Acquisition of English Thematic Roles by College Students: Focusing on the English Verb Openl presented at International Symposium on Advanced and Applied Convergence held on November 1416, 2013 at Seoul, Korea. We would like to express our sincere gratitude to al the Chairs and the members of Editorial Committee of the 1st International Symposium on Advanced and Applied Convergence \& Advanced Culture Technology.

\section{References}

[1] R. Berwick, "The Acquisition of Syntactic Knowledge” Cambridge, Mass.: MIT Press, (1985).

[2] N. Chomsky, "Lectures on Government and Binding" Foris Dordrech (1981).

[3] N. Chomsky, "Knowledge of Language", Praeger New York, (1986).

[4] N. Chomsky, "Some Notes on Economy of Derivation and Representation", MITWPL, vol. 10, (1989).

[5] N. Chomsky, "The Minimalist Program”, Cambridge, Mass. MrT Press, (1995).

[6] N. Chomsky, "Minimalist Inquires: The famework", Roger Martin, David Michaels, and Juan Uriagerreka (eds) Step by Step: Essays on Minima ist Syntax in Honor of Howard Lasnik, Cambridge, Mass: MIT Press, (2000), pp. 89-155.

[7] N. Chomsky, "Derivation by Phrase Michael Kenstowicz (eds) K. Hale: A Life in Language, Cambridge, Mass: MIT Press, (2001), pp 1-52.

[8] N. Chomsky, Problems of Projection. Lingua, vol 130, (2013), pp. 33-49.

[9] D. Crystal, “A Dictionary of Linguistic and Phonetics”, Blackwell, Oxford, (1996).

[10] D. R. Dowty, "Thetmatic Proto-roles and Argument selection”, Language, vol. 67, (1991), pp. 574-619.

[11] R. Ellis, "The Study of Second Language Acquisition”, Oxford University Press, (2012).

[12] R. Ellis, Second anguage Acquisition. Oxford University Press, (2012).

[13] C. J. Fillm ore, "The Case for Case", In E. Bach and R. Harms (eds) Universals in Linguistic Theory, New York: Holt, Rinehart \& Winston, (1968), pp. 1-88.

[14] T. Givón, "Direct Object and Dative Shifting: Semantic and Pragmatic Case", Frans Plank (ed.) Objects: towards a theory of grammatical relations, London: Academic Press, (1984) pp. 151-82.

[15] J. Grimshaw, “Argument Structure”, Linguistic Inquiry Monographs 18. Cambridge, MA: MIT press, (1990).

[16] N.-K. Kang and H. Morita, "The Acquisition of English Thematic Role by College Students: Focusing on the English Verb Open”, Proceedings of 1st International Symposium on Advanced and Applied Convergence \& Advanced Culture Thechnology, Jeong Jin Kang, Edward J. Rothwell, Yanghao, and Byungjoo Park (eds), (2013), pp. 163-169.

[17] R. K. Larson, “On the Double Object Construction”, Linguistic Inquiry, vol. 19, (1988), pp. 335-91.

[18] F. R. Palmer, "The English Verb”, Longman Publishers, (1987).

[19] A. Radford, "Transformational Syntax: A Student's Guide to Chomsky's Extended Standard Theory", Cambridge University Press, (1981).

[20] G. Ramchand, "Verb Meaning and the Lexicon", Cambridge University Press, (2010).

[21] H. Ringbom, "The Importance of Cross-linguistic Similarity in Foreign Language Learning: Comprehension, Learning and Production", Clevedon: Multilingual Matters, (2007).

[22] J. I. Saeed, "Semantics”, Wiley-Blackwell, (2009).

[23] J. Schachter, "A New Account of Language Transfer in S. Gass and L. Selinker", Language Transfer in Language Learning. Rowley, Mass: Newbury House. 


\section{Appendix}

If the following sentences are grammatical, write "grammatical" in the brackets. If the following sentences are not grammatical, write "ungrammatical" in the brackets.

1. a. The door opened . [ ] ]

b. ku mwun-i yellessta. [

2. a. The boy opened the door. [

b. Ku sonyen-i ku mwun-ul yelessta.

3. a. The key opened the door. [ ]

b. Ku ki-ka kumwun-ul yelessta. [

4. a. The boy opened the door with the key. [

b. Ku sonyen-un ku mwun-ul ku kilo yelessta. [

c. Ku sonyen-un ku kilo ku mwun-ul yelessta. [

5. a. The key opened. [

b. Ku ki-ka yelessta. [

6. a. The boy opened. [

b. Ku sonyen-i yelessta. [

7. a. The door opened with a key. [

b. Ku mwun-i kilo yellessta. [

8. a. The door opened by the boy. [

b. Ku mwun-i sonyen-euyhay yellessta. [

9. a. The key opened the door by the boy . I

b. Ku ki-ka ku mwun-ul ku sonyen-eu

c. Ku ki-ka ku sonyen-euyhay ku mw un-ul yelessta.

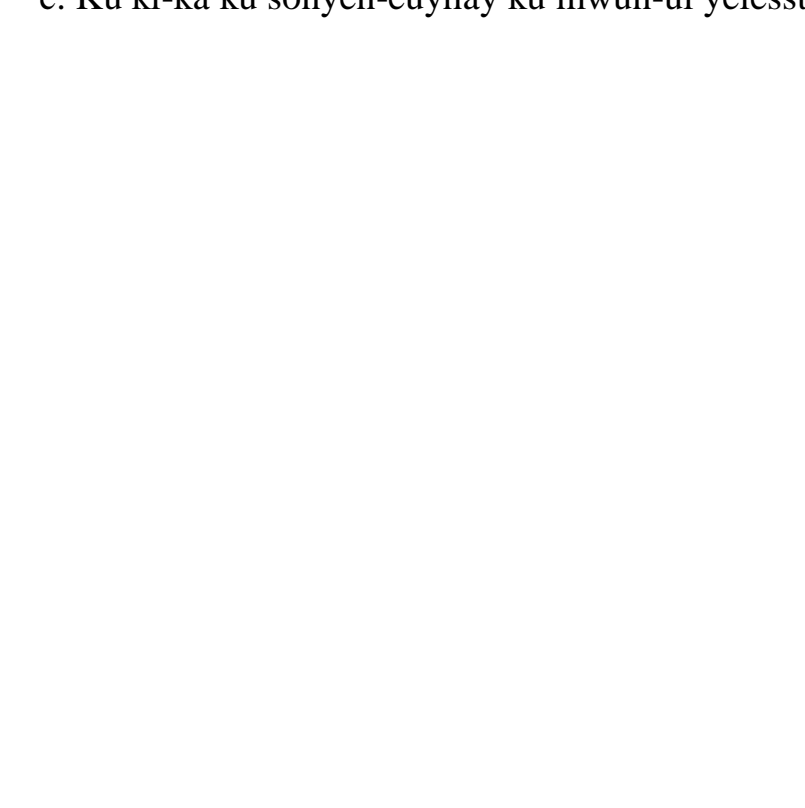


International Journal of Smart Home

Vol.8, No.2 (2014)

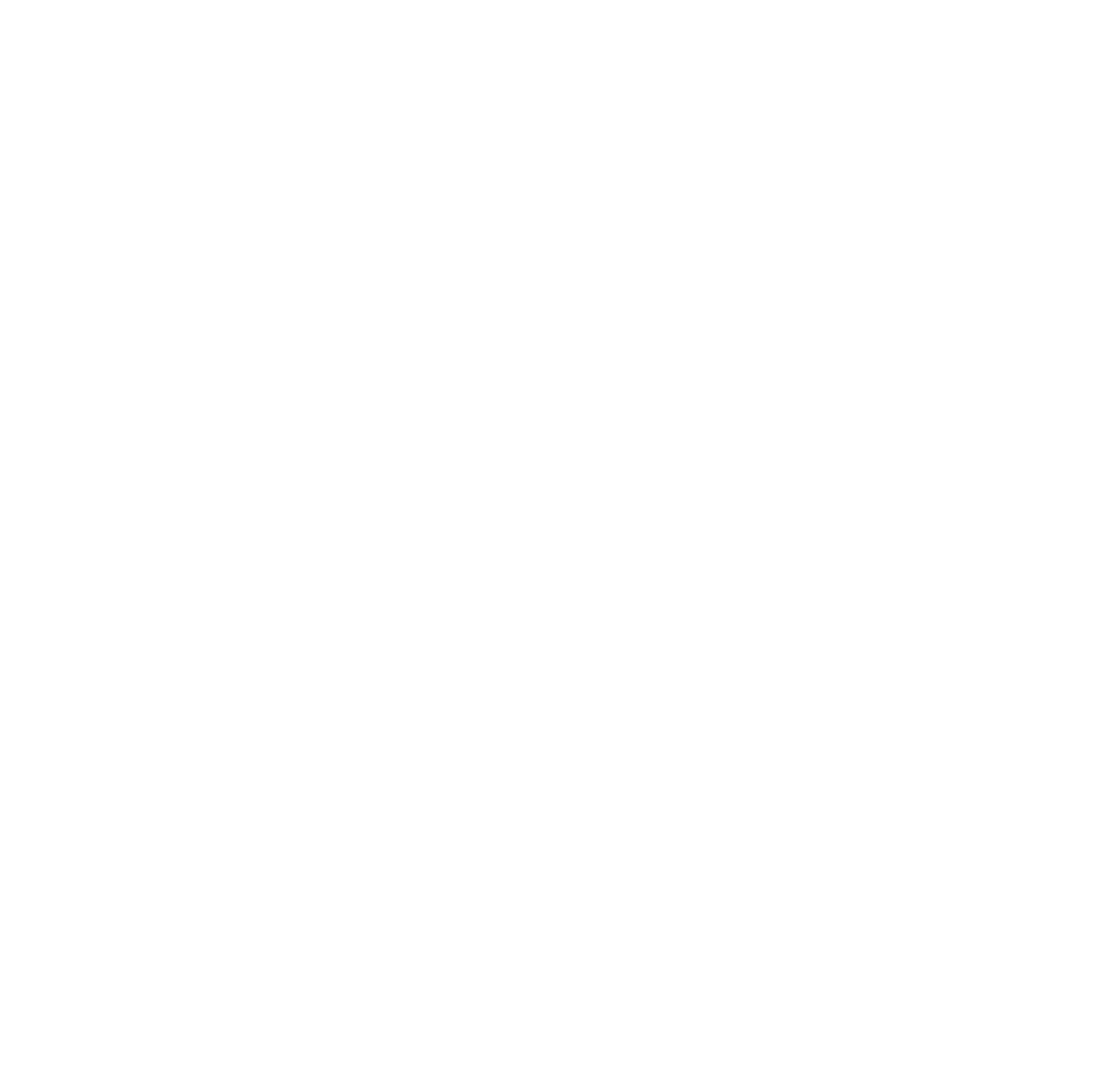

